\title{
Transverse Flux Linear Machine with High Thrust for Direct Drive Applications
}

\author{
Junghwan Chang ${ }^{1 *}$, Jiwon Kim², Dohyun Kang' ${ }^{2}$ and Deokje Bang ${ }^{3}$ \\ ${ }^{1}$ Department of Electrical Engineering, Dong-A University, Busan 604-714, Korea \\ ${ }^{2}$ Electric Motor Research Center, KERI, Changwon 641-120, Korea \\ ${ }^{3}$ Electrical Power Processing Lab, Delft University of Technology, Delft, The Netherlands
}

(Received 10 April 2010, Received in final form 26 May 2010, Accepted 27 May 2010)

\begin{abstract}
This paper describes the development of a novel transverse flux linear motor (TFLM) excited by permanent magnets (PMs). It combines the advantage of two different TFLMs and produces high thrust with reduced normal force. The magnetic field is analyzed by combining the three-dimensional (3D) equivalent magnetic circuit network (EMCN) method with 2D finite element analysis. The experimental findings of the prototype motors are in good agreements with the analysis results, and demonstrate the potential of the proposed motor as a direct drive requiring relatively long displacement of a mover.
\end{abstract}

Keywords : direct drive, high thrust, linear motor, transverse flux

\section{Introduction}

In direct drive applications, the main issue is to increase the force and power density of the machine by improving the constructional design of the stator and mover. This normally requires careful consideration in the design of the magnetic topologies, which promotes significant weight reduction in the magnetic mass as well as improvement in the form factor of the magnetic design [1]. Among the several novel forms of permanent magnet (PM) machines that have been developed for such applications, transverse flux machines are recognized as a candidate to achieve the highest force density, especially at a low speed.

Transverse flux linear motors (TFLMs) with PM excitation have been devised by combining high-energy PMs, flux-concentrating magnetic circuit design, and threedimensional (3D) flux flow. Relative to other motors, they have a number of useful features [2-4]. Most importantly, there is no competition between the space requirement of the flux carrying core irons and the space occupied by armature windings. This increases the design flexibility compared with the longitudinal flux machines, in which there is a fundamental conflict between electric and magnetic loading. The other benefit of the TFLMs with PM excitation is their ability to increase the force density

*Corresponding author: Tel: +82-51-200-7735

Fax: +82-51-200-7743, e-mail: cjhwan@dau.ac.kr with increasing pole number or decreasing pole pitch for given geometrical dimensions. With these benefits, it is commonly accepted that the TFLMs with PM excitation offer a very high force density compared with other machine types [5]. Therefore, TFLMs with PM excitation seem to be suitable for direct drive applications requiring high force density.

In this research, two different kinds of TFLM with PM excitation are compared, and a novel transverse flux machine is suggested by combining the advantages of the two structures. The magnetic field is analyzed by 3D equivalent magnetic circuit network (EMCN) method using a symmetry condition of flux distribution. 2D finite element analysis is also used to determine the influence of the end effect of the mover on the TFLM performance. The experimental results of the prototype motors are in good agreements with the analysis ones, and the newly developed motor seems to be especially suited for direct drive applications requiring high force density.

\section{A Novel Transverse Flux Machine}

\subsection{Conventional design review}

Fig. 1 shows the single phase of two different TFLMs with PM excitation that have been presented in our previous research [6]. In each mover, the PMs are fitted between the iron cores and magnetized with alternating polarity facing to each other. This arrangement produces a 
high air gap flux density in the air gap region compared to other PM motor types. The ends of the mover are skewed by one pole pitch relative to each other, and form the path for 3D flux flow with the adjacent teeth of the stator. These features produce unidirectional thrust at the entire region by reversing the polarity of the applied current.

The major differences between the models are the positions of the armature windings and the presence of normal force. The first model has armature windings in the stator and a mover with I-shaped cores (I-MC) is inserted between the poles of the stator. In this case, there is no normal force and the lateral forces existing in the two air gaps between the teeth of the stator and the mover cancel each other out. However, some applications require long mover stroke, and it is not practical to mount armature windings in the long stator considering the effective utilization of windings and the significant losses of magnetic flux. The second model has U-MCs and the armature windings are inserted into the legs of the mover. This feature is suitable for applications requiring relatively long displacement of the mover compared to the I-MC. However, this motor type produces a much bigger normal force than the thrust, which limits its use due to the friction losses caused by the normal force.

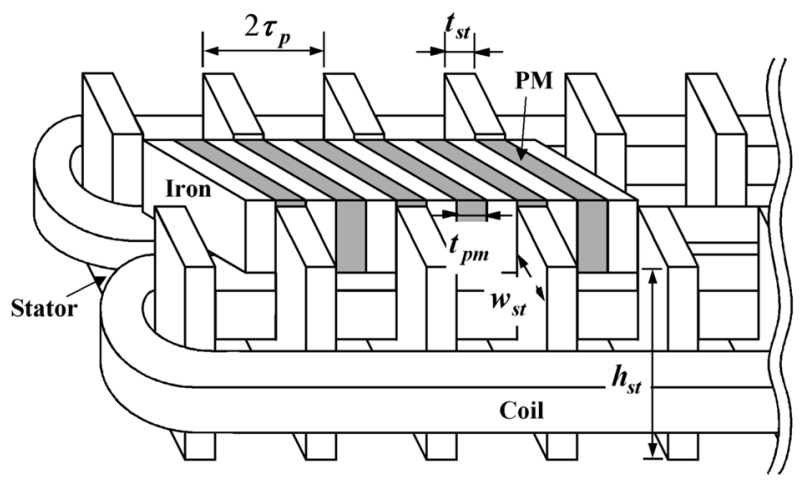

(a)

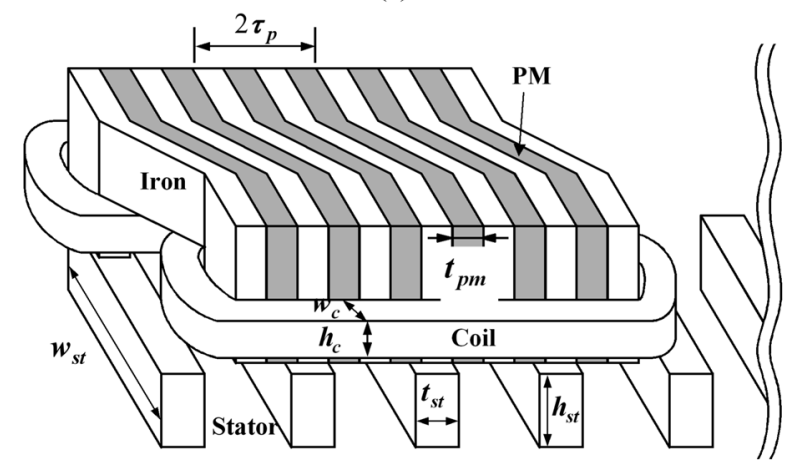

(b)

Fig. 1. Conventionally designed TFLMs: (a) I-MC, and (b) UMC.

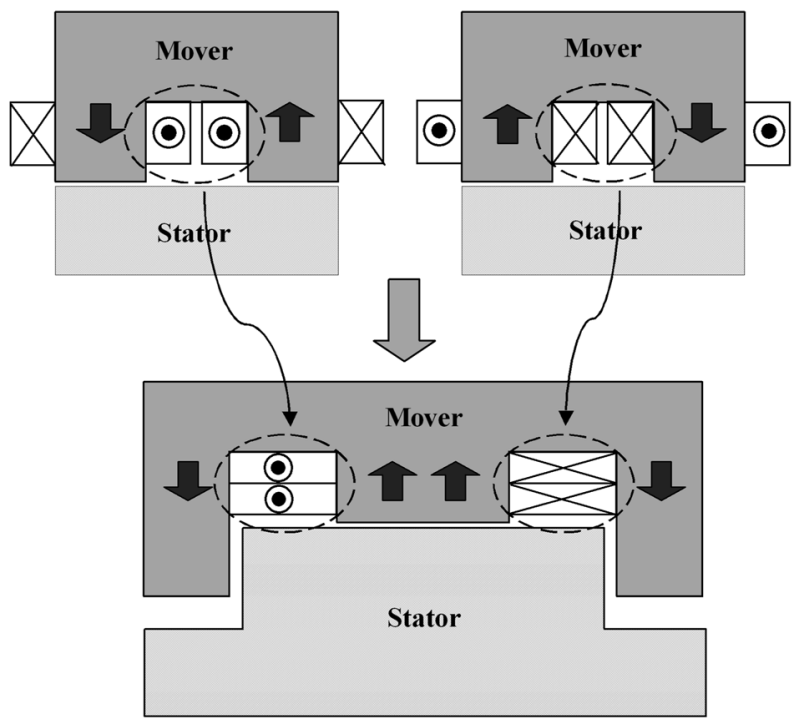

Fig. 2. Development process for the new TFLM.

\subsection{Novel design (E-MC)}

The stocker system, which is used for transferring glasses in the LCD industry, needs linear direct drives to avoid drawbacks of traditional drives with rotary motor and mechanical motion conversion devices. In this application, the stator is installed up to $100 \mathrm{~m}$ and system requires a thrust of $10,000 \mathrm{~N}$. Thus, it is difficult to apply the I-MC alone due to the flux leakage caused by the long stator. Of course, the U-MC can be applied to this application, but it has also suffers the drawback of a huge normal force.

In the conceptual development process shown in Fig. 2, the new E-MC model is developed by combining the advantage of the previous two models; the non-existence of a normal force of the I-MC and the effective coil arrangement of the U-MC. Basically, it can produce twice the thrust of the U-MC by jointing it in a parallel way. However, the normal force is increased by less than $50 \%$ compared with the U-MC, because the extruding pole arrangement of the newly designed E-MC at both ends of the mover provides only thrust, and the attractive forces between the stator and mover cancel each other out. The other advantage of the E-MC is the amount of coppers windings used, which are reduced by almost $50 \%$. This implies that the winding losses of the E-MC can be substantially decreased comparing with the other two types. Fig. 3 shows the single phase of the E-MC TFLM.

\section{Analysis and Experiment Results of TFLMs}

\subsection{Analysis results}




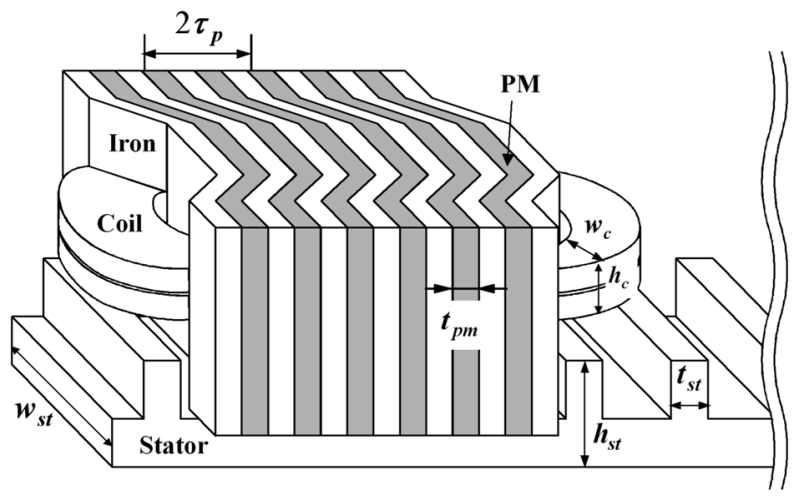

Fig. 3. Newly designed TFLM having E-MC.

Table 1. Specifications of the designed E-MC TFLM motors.

\begin{tabular}{llll}
\hline \multirow{2}{*}{ Symbol } & \multicolumn{2}{c}{ Quantity } \\
\cline { 2 - 4 } & \multicolumn{1}{c}{ U-MC } & \multicolumn{1}{c}{ E-MC } \\
\hline DC link voltage & $300[\mathrm{~V}]$ & $300[\mathrm{~V}]$ \\
Number of turns & 288 turns & 160 turns \\
Rated MMF & $5000[\mathrm{~A} \cdot \mathrm{T}]$ & $5000[\mathrm{~A} \cdot \mathrm{T}]$ \\
Air gap length & & $2.0[\mathrm{~mm}]$ & $2.0[\mathrm{~mm}]$ \\
& $\tau_{p}$ & $20[\mathrm{~mm}]$ & $20[\mathrm{~mm}]$ \\
Stator & $t_{s t}$ & $14[\mathrm{~mm}]$ & $14[\mathrm{~mm}]$ \\
& $w_{s t}$ & $130[\mathrm{~mm}]$ & $260[\mathrm{~mm}]$ \\
& $h_{s t}$ & $40[\mathrm{~mm}]$ & $74[\mathrm{~mm}]$ \\
\multirow{2}{*}{ Mover } & $t_{p m}$ & $10[\mathrm{~mm}]$ & $10[\mathrm{~mm}]$ \\
& $w_{c}$ & $20[\mathrm{~mm}]$ & $50[\mathrm{~mm}]$ \\
& $h_{c}$ & $30[\mathrm{~mm}]$ & $40[\mathrm{~mm}]$ \\
Material & Stator & S23 & \\
& mover & SM490A & \\
\hline
\end{tabular}

To compare the performance of the U- and E-MCs, each model is designed and its geometrical dimensions and physical properties are shown in Table 1. Fig. 4 presents the B-H curves of the used magnetic materials. Unlike the rotating machines, linear motors like TFLM experience an end effect due to the open characteristics of the magnetic circuit at the entry and exit ends of the mover. However, it is not practical to analyze the TFLM with whole 3D model to consider both 3D flux flow and leakage and fringing flux at the ends of mover. Therefore, as our previous studies [6, 7], the magnetic field of each model is analyzed by combining the results of the two parts of the analysis.

One analysis part is the $3 \mathrm{D}$ analysis by EMCN method. This represents the analysis region by the connection of the permeance elements and is based on the continuity of the magnetic flux at each node. The conceptual model of the equivalent magnetic circuit at a node is given in

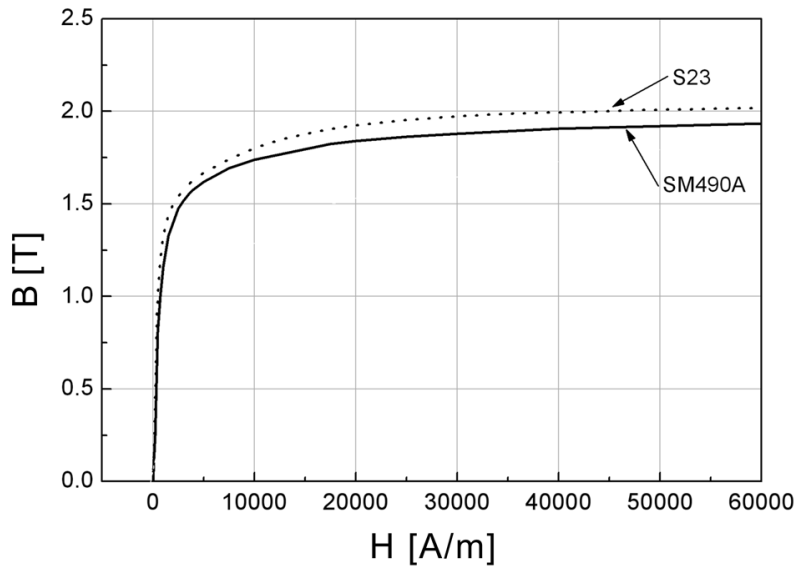

Fig. 4. B-H curves of the used magnetic materials.

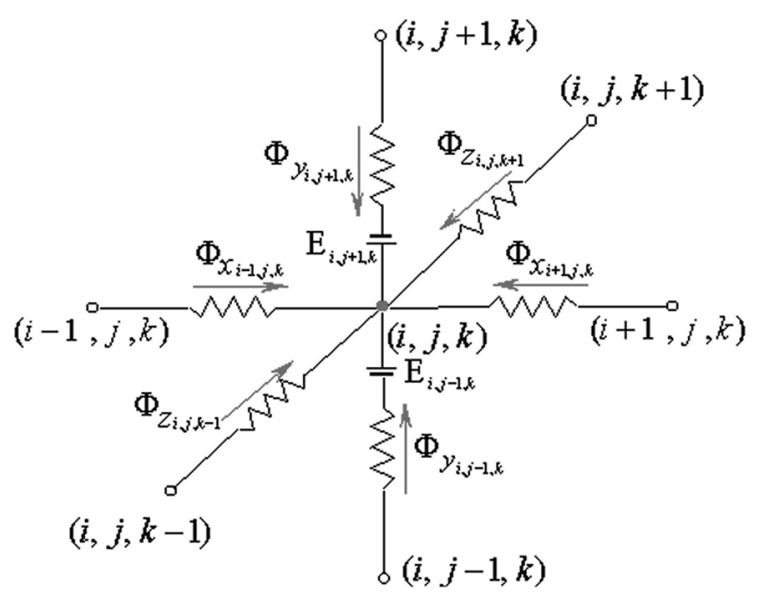

Fig. 5. Conceptual model of equivalent magnetic circuit.

Fig. 5. The sum of the magnetic flux flowing into that node is equal to the sum of magnetic flux flowing out of that node. This can be stated as follows by considering a total of 6 branches with magnetic flux flowing in each direction.

$$
\begin{aligned}
\Sigma \Phi_{\text {node }}= & \Phi_{x, i-1, j, k}+\Phi_{x, i+1, j, k}+\Phi_{y, i, j-1, k}+\Phi_{y, i, j+1, k} \\
& +\Phi_{z, i, j, k-1}+\Phi_{z, i, j, k+1}
\end{aligned}
$$

Each magnetic flux in (1) can be calculated with the permeance between nodes, magnetic scalar potential at each node and the equivalent magnetomotive force (MMF) by external sources. Finally, we obtain the system matrix (2).

$$
[P]\{U\}=\{F\}
$$

where, $[P]$ is the permeance coefficient matrix, $\{U\}$ the vector of the magnetic scalar potential and $\{F\}$ the forcing vector [8].

With the symmetry condition of flux distribution, the 


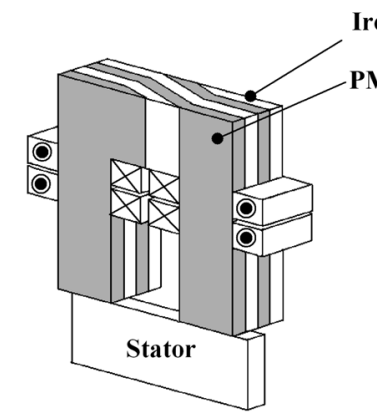

(a)

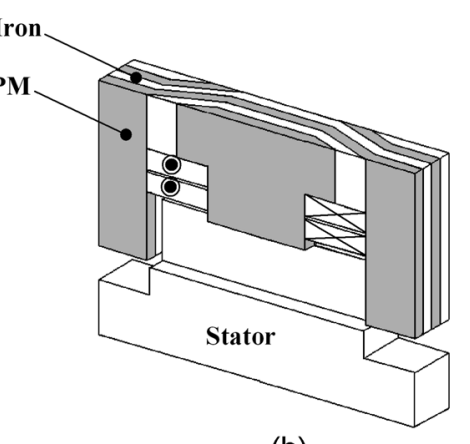

(b)
Fig. 6. Analysis model for the EMCN method: (a) U-MC, and (b) E-MC.

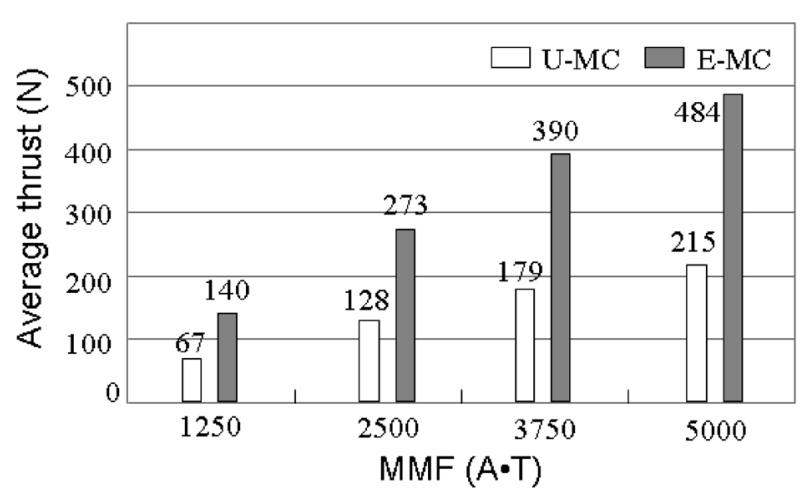

(a)

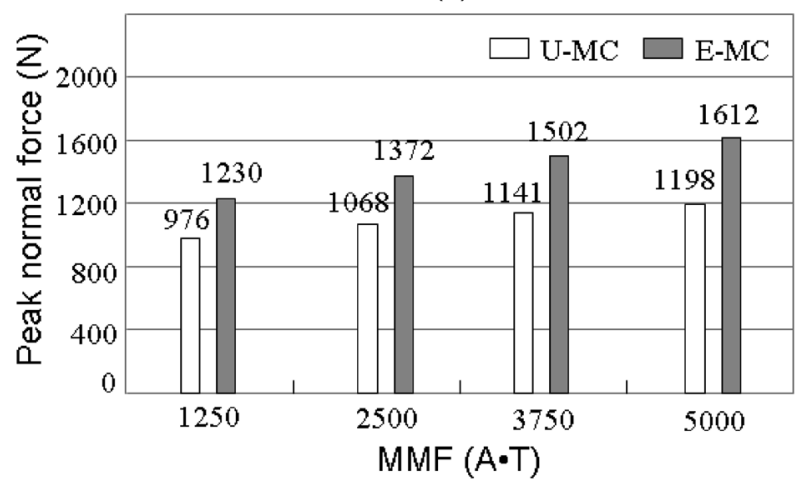

(b)

Fig. 7. Comparison of thrust and normal force: (a) average thrust, and (b) peak normal force.

analysis model of each type is one part of the total model, corresponding to one pole pair of the mover, as shown in Fig. 6. The other analysis part is the $2 \mathrm{D}$ finite element analysis of the equivalent magnetic circuit model to determine the influence of the end effect of the mover on the TFLM performance. The two incomplete poles at the both ends of the mover reduce the average thrust by $40 \%$ compared with the other pole pairs located at the insides of the mover $[6,7]$.
The performance of the entire machine can be obtained by considering both results. The U-MC has 16 -iron cores and 15-PMs and the E-MC has 20-iron cores and 19-PMs with iron cores at the both ends of the movers. Therefore, each prototype motor can be considered to have 7 and 9 complete pole pairs and two incomplete poles at the entry and exit regions of the mover. The magnetic force due to the complete pole pairs can be obtained by multiplying the number of pole pairs by the $3 \mathrm{D}$ analysis results, while the magnetic force due to the both ends of the mover can be considered by multiplying the 3D results by a correction factor of 0.6. The total magnetic force is the sum of these two values.

Fig. 7 shows the thrust and normal forces of TFLMs having U- and E-MCs. For comparison, each value corresponds to one-pole pair of each model when applying different MMF magnitudes.

The E-MC produces over twice the thrust of the U-MC at all applied MMFs. At $50 \%$ and $100 \%$ of the rated MMF, the average thrust of the E-MC TFLM is 2.13 and 2.25 times higher than that of the U-MC TFLM, respectively. The force producing area and total volume of the E-MC are twice those of the U-MC. Thus, comparing the thrust density at the rated MMF, the E-MC has a $12.5 \%$ higher thrust density than that of the U-MC.

However, the peak amplitude of the normal force of the E-MC is only increased by about $30 \%$ compared with that of the U-MC due to the cancellation of the attractive force between the mover and stator at the both sides of the mover in the E-MC. The ratio of peak normal force to average thrust decreases with increasing applied MMF, as shown in Fig. 8. However, even in the E-MC, the peak value of the normal force is about three times higher than the average thrust at the rated MMF. This will increase friction loss and affect the dynamic behavior of the motor. Therefore, it is important to design a magnetic circuit

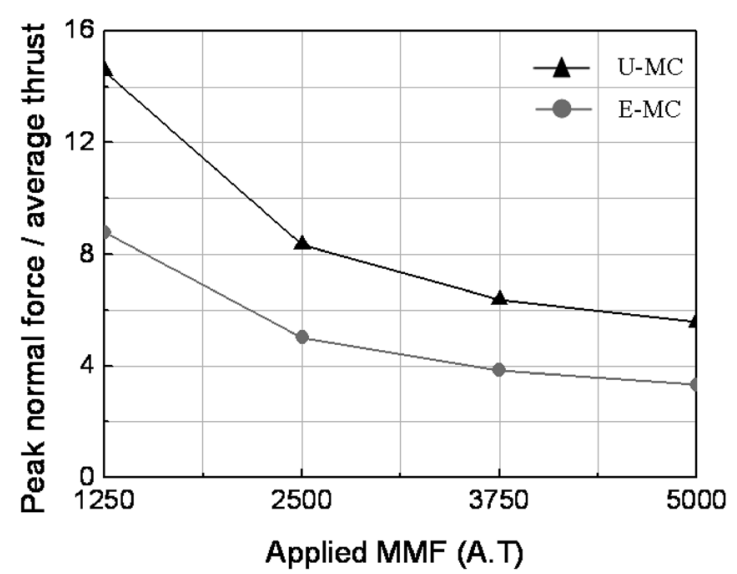

Fig. 8. Peak normal force to average thrust ratio. 
reducing normal force without sacrificing other characteristics such as the thrust and volume of the motor.

\subsection{Experimental results}

Figs. 9 and 10 show the movers and stators of the Uand E-MC TFLM for static experiments, respectively. Each pole of the mover is skewed by one pole pitch to generate a unidirectional force at the entire region, whereas the stator has a straight path for magnetic flux. They all have flux-concentrating structures with PMs excitations and single-sided topologies.

For the experiment, the stator and mover of each prototype motor are installed with a predefined air gap length and the thrust is measured with load cells at each displacement of the mover according to the variation of the applied current, as shown in Fig. 11.

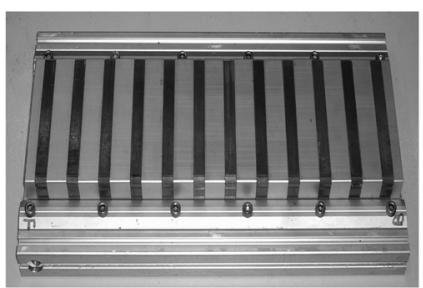

(a)

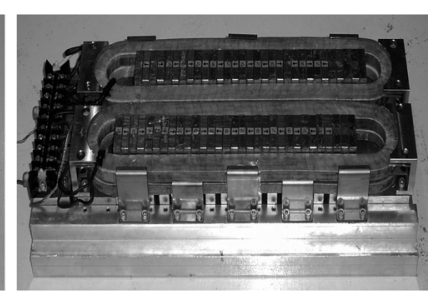

(b)
Fig. 9. U-MC TFLM: (a) stator, and (b) mover.

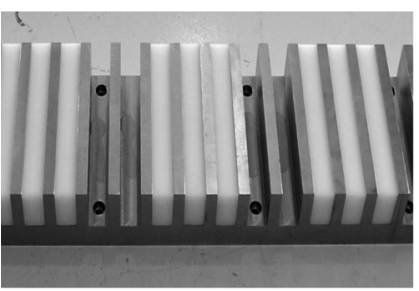

(a)

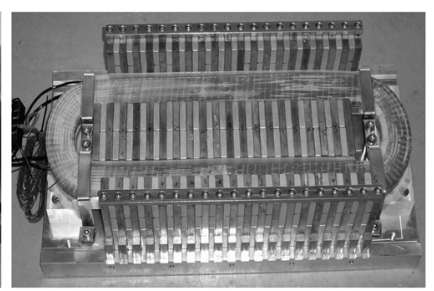

(b)
Fig. 10. E-MC TFLM: (a) stator, and (b) mover.

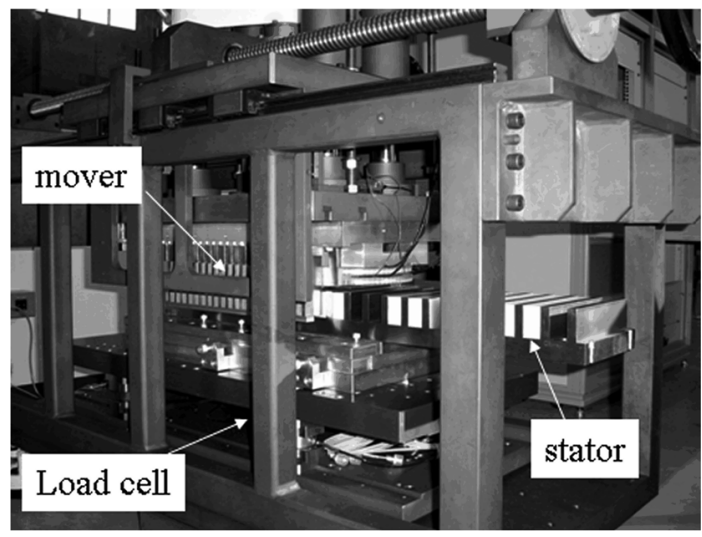

Fig. 11. Experiment setup for measuring forces.

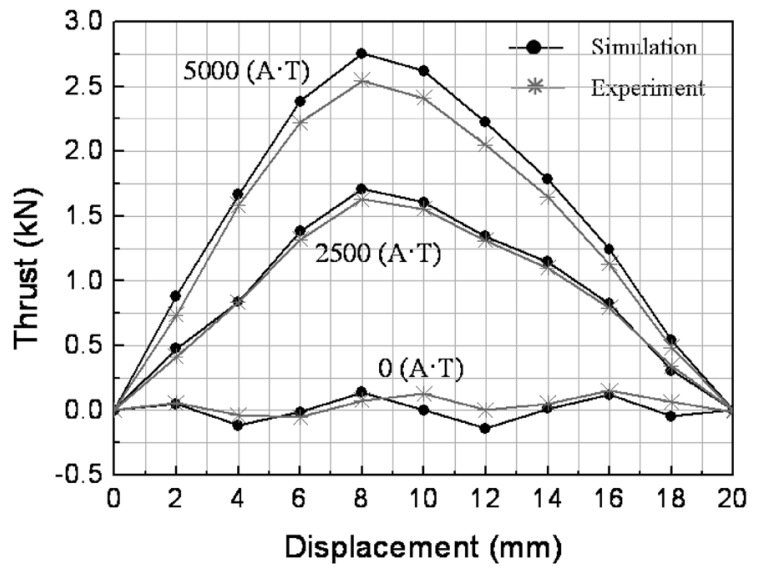

(a)

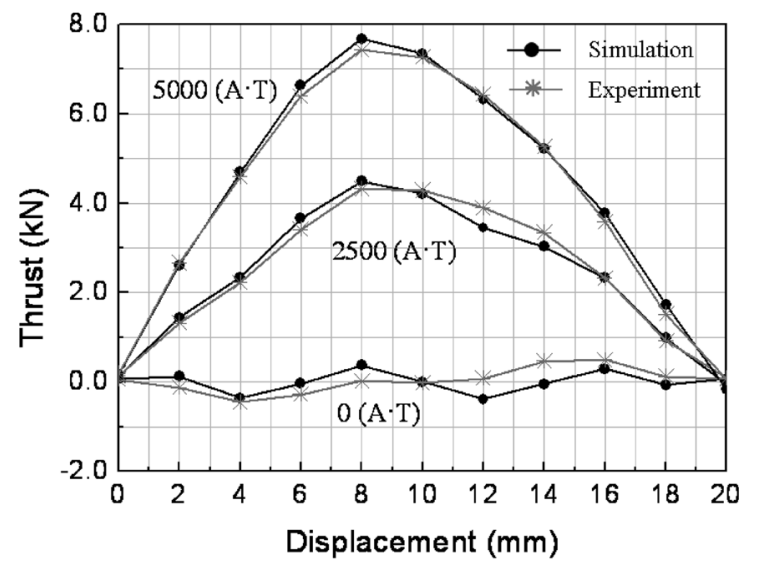

(b)

Fig. 12. Thrust force profile: (a) U-MC, and (b) E-MC.

Fig. 12 compares the thrust profiles for different MMFs during the displacement of the mover between the stable and unstable equilibrium positions. The average thrusts are in good agreement with experimental ones. At 50\% and $100 \%$ of the rated MMFs, the experiment shows that the average thrust of the E-MC is 2.29 and 2.43 times higher than that of the U-MC, respectively.

\section{Conclusions}

This research deals with the development of a novel TFLM having E-MCs for direct drive applications. The newly designed model produces twice the thrust of the TFLM with U-MCs by combining them in parallel. However, it only offers a small increase in the normal force, and the amount of copper for the windings can be reduced by almost $50 \%$ of that of the U-MCs. The analysis findings are verified by the experimental results, which demonstrate that the newly proposed E-MC TFLM motor can be used for direct drive applications requiring relatively long displacement of the mover such as stocker 
systems and ropeless elevators.

\section{References}

[1] Z. Soghomonian, Z. Rahman, and K. Martin, 23rd Annual Conference on Properties and Applications on Magnetic Materials (2004).

[2] W. M. Arshad, T. Backstrom, and C. Sadarangani, IEMDC 115 (2001).

[3] G. Henneberger and M. Bork, IEE Colloquium on New Topologies for PM Machines (1997).
[4] Waqas Mahmood Arshad, Doctoral dissertation, Royal Institute of Technology, Stockholm (2003).

[5] A. J. Mitcham, IEE Colloquium on New Topologies for PM Machines (1997).

[6] Junghwan Chang and Dohyun Kang, Journal of Electromotion 12, 99 (2005).

[7] Junghwan Chang, Dohyun Kang, Jiyoung Lee, and Jungpyo Hong, IEEE Trans. Magn. 41, 1936 (2005).

[8] V. Ostovic, Dynamics of Saturated Electric Machine, Springer-Verlag, Heidelberg (1989). 